\title{
The Quest for High Quality, Ultra-Safe Care in Pulmonary/Respiratory Medicine
}

\section{Frank Mazza*}

VP / Chief Patient Safety Officer / Associate Chief Medical Officer, Seton Healthcare Family, 1345 Philomena Street, Suite 410.3, Austin, TX 78723, USA

I have never seen a survey of pulmonary/respiratory (or other healthcare) professionals that asks what degree of excellence they aspire to in their delivery of care, and I cannot fathom that I ever will. It is unimaginable to me that in a hypothetical Likert scale query of physicians in practice or training, any would ever seriously answer that they intend that anything but the highest quality and the safest care be delivered on behalf of their patients. And yet studies of our clinical systems and processes and of patient care across the globe relate an inconvenient truth. The Dartmouth Atlas' chronicles of the stunning variation in care throughout the United States (with no correlation to quality for the cost) and the estimated 44,000 to 98,000 deaths per year due to medical errors referenced in the IOM report "To Err is Human" sadly serve to help validate this alternative reality.

So where is the disconnection between the fervent dedication of healthcare professionals and the reality of the suboptimal outcomes that we have all come to know that characterize healthcare? We can all recite numerous reasons - there is the sheer complexity of healthcare across all its broad domains and among all its moving parts. As a complexadaptive system, there is little to rival healthcare in terms of the need to coordinate all aspects of outpatient and inpatient care or the multiple service lines within the hospital (such as the ICU, ED, OR, the clinical laboratory and imaging areas, pharmacy, and admissions to name a "few".)And of course, there is the dramatically and rapidly expanding evidence base that no human could ever reasonably be expected to commit to memory in even a reasonable fraction of its entirety. At the center of all this is the patient, a notable variable herself in terms of her manifestations of disease and in her response to therapy. A pessimist could be tempted to say that there are too many complex pieces to the system, too much variation in clinical presentation and response, and too incomprehensible a repository of knowledge to ever expect that anything even close to the highest quality and the safest care could ever be expected to be delivered every time to every patient. At least for the present, there appears to be some validity to that statement.

But in the realm of the possible, the reality of the present should not discourage our efforts to continually improve the care that we deliver, nor has it ever done so in the past. We have only to look at the striking number of new drugs, the panoply of medical devices, the robust treatment protocols and the care delivery models that have emerged over the last fifty years that we can point to with pride in affirming that the quest for medical progress remains front and center for scientists and practitioners alike.

For sure, we can't solve all of the complex and sub-optimal performance issues that characterize our healthcare system at one time. But is there is a better way for us to direct our efforts that promises to accelerate improvements in the quality of care we deliver and to responsibly steward our limited resources in the process? I think there is.

We have a long heritage of focusing on and incrementally uncovering a better understanding of basic medical science, whether at the organsystem, cellular or sub-cellular level. It is critical that this work continue. In addition, the beauty of the capital markets is that, in the end, the incentive to develop safer and more effective pharmaceuticals and to design more powerful and more innovative medical products benefits both the innovator and the recipient of medical care. For all the rightful worries of how we will pay for them in the future, both the predictable and unimaginable technological advances that we will experience offer great and wonderful promise.

In my mind, the biggest "bang for the buck" in improving the quality and safety of care lies in the realm of human factors and high reliability science. When you introduce a new protocol for managing patients with community acquired pneumonia, you provide a worthy service for patients with CAP in your practice or at your local hospital. But when you teach your staff how to function as a team, or you flatten the medical hierarchy, decreasing the power distance between providers, you potentially affect care across a much wider domain. Staff feel empowered to speak up rather than to avoid questioning the surgeon who is about to remove the wrong lung because he is known to be difficult and unapproachable. Problems are more reliably and more comprehensibly solved when all providers have the opportunity to plan and debrief after events and to re-engineer suboptimal processes in a systems context. The skill set and psychological safety needed to effectively do this across the multiple domains of care works well when we see peer coaching, peer checking and behavioral accountability on the part of all. This approach can be applied to our OR, ICU, ED, MedSurg units as well as our housekeeping, facility and kitchen departments, all at the same time.

So do we have any hope of getting there anytime soon? The ACGME and many specialty societies clearly understand the need to solve problems in a systems context. More and more hospitals and their medical staffs are focusing on the risks associated with human factors and are learning of the virtues of 'high reliability.' But there are still pockets of resistance- the long held belief that physician autonomy must be vigorously defended because it is good for patients must be respectfully questioned and subjected to scientific scrutiny. Thankfully, high reliability and human factors science is highly evidence-based and intrinsically makes sense to clinicians and non-clinicians alike. We need to convince physicians to take time out from their busy practice to experience and learn the guiding principles and to persuade hospitals that the dedication of time and resources for staff training is worthy of the "non-productive time" it exacts. If we mean what we all say- that quality and safety come first-we will find the time and resources to do this. In the end, I believe that we will all be better off for the effort.

*Corresponding author: Frank Mazza, MD, VP / Chief Patient Safety Officer Associate Chief Medical Officer, Seton Healthcare Family, 1345 Philomena Street, Suite 410.3, Austin, TX 78723, USA, Tel: (512)-423-1732; Fax: (512)-380-7556; E-mail: fmazza@seton.org

Received March 18, 2012; Accepted March 20, 2012; Published March 22, 2012

Citation: Mazza F (2012) The Quest for High Quality, Ultra-Safe Care in Pulmonary/ Respiratory Medicine. J Pulmonar Respirat Med 2:e111. doi:10.4172/2161105X.1000e111

Copyright: (C) 2012 Mazza F. This is an open-access article distributed under the terms of the Creative Commons Attribution License, which permits unrestricted use, distribution, and reproduction in any medium, provided the original author and source are credited. 